\title{
Las restricciones a la libre circulación de personas en la UE durante la era COVID-19: hacia un futuro incierto
}

\author{
Restrictions on the Free Movement of \\ Persons in the EU During the COVID-19 \\ Era: Towards an Uncertain Future
}

\author{
Magdalena M. Martín ${ }^{1}$ \\ Universidad de Málaga (España)
}

Recibido: 13-09-20

Aprobado: 11-10-20

\section{Resumen}

Este estudio analiza el impacto que la COVID-19 ha supuesto para la libre circulación de personas y el control de las fronteras tanto interiores como exteriores en la UE, en un momento especialmente delicado, ya que ha coincidido con la apertura de la Conferencia sobre el futuro de la UE. La hipotésis de partida es la inadecuación del marco jurídico de la libre circulación, tanto en el Derecho Internacional como en el Derecho Europeo, para adoptar las restricciones que la lucha contra la pandemia exige. Este hecho, unido a que los Estados miembros son competentes en materia de salud pública y en la preservación de su seguridad nacional, han alterado la esencia y los límites de un derecho clave para el presente

\footnotetext{
${ }^{1}$ (magdalena@uma.es). Catedrática de Derecho Internacional Público y Relaciones Internacionales en la Universidad de Málaga. Doctora en Derecho por el Instituto Universitario Europeo de Florencia. Ha impartido conferencias y seminarios en las principales Universidades de España (Madrid, Barcelona, Sevilla, Salamanca, Santiago de Compostela etc.) y en Iberoamérica (Costa Rica, Perú, Venezuela). En la actualidad sus principales líneas de investigación tienen que ver con el Espacio Europeo de Libertad, Seguridad y Justicia y la acción exterior de la Unión Europea, la inmigración y el género, y el Derecho Internacional Penal. Entre sus publicaciones más recientes destacan: Límites a la libre circulación de personas en la UE por razones de orden público, seguridad o salud pública en tiempos de crisis: una revaluación a la luz de la jurisprudencia del TJUE, Revista de Derecho Comunitario Europeo, Año $\mathrm{n}^{\circ}$ 18, No 49, 2014; La crisis de los refugiados sirios como amenaza a la seguridad internacional y europea: paisaje después del naufragio, en "Hacia una identidad europea en materia de seguridad y defensa: ¿realidad o utopía?” (coord. por Andrés Bautista Hernáez, Alicia María Pastor García; Elena del Mar García Rico (dir.), María Isabel Torres Cazorla (dir.), 2019; La enésima refundación de la UE: el dilema indefectible sobre el modelo y la esencia de la futura Europa en Principios y justicia en el derecho internacional, en libro homenaje al profesor Antonio Remiro Brotóns, coord. por Carmen Martínez Capdevila, Irene Blázquez Navarro; Javier Díez-Hochleitner Rodríguez (ed. lit.), Carlos Espósito Massicci (ed. lit.), Cristina Izquierdo Sans (ed. lit.), Soledad Torrecuadrada García-Lozano (ed. lit.).

ORCID: https://orcid.org/0000-0002-8080-4847.
} 
y el futuro del proceso de integración europea. De ahí que sea necesario preservar el actual acervo normativo frente a estas y otras posibles derivas restrictivas, evitando así dar por sentado su irreversibilidad.

Palabras-clave: COVID-19, Libre circulación de personas, control de fronteras, restricciones, levantamiento, Conferencia sobre el Futuro de la UE.

\begin{abstract}
This study analyzes the impact that the COVID-19 has had on the free movement of people and the control of both internal and external borders in the EU, at a particularly delicate time, coinciding with the opening of the Conference on the future of the EU. The hypothesis is that the legal framework of free movement both in International Law and in European Law is not suitable to adopt the restrictions that the fight against the pandemic requires. This fact, in addition that public health and the preservation of national security are the competences of the Members States, have altered the essence and limits of a key right for the present and the future of the process of European integration. Therefore, the normative heritage developed by the EU in this matter must be preserve against these and other restrictive drifts, avoiding taking its irreversibility for granted.
\end{abstract}

Key-words: COVID-19, Free Movement of People-Border Control, Restrictions, Lifting, Conference on the Future of the EU.

\title{
1. Consideraciones preliminares
}

La pandemia sanitaria provocada por el coronavirus, y la macro crisis sin precedentes que ha traído aparejada, han marcado un antes y un después en las relaciones individuales y colectivas. Ya sea como personas físicas, como nacionales de un Estado, o en tanto que ciudadanos europeos en un mundo global, nuestro modo de vida se ha visto profundamente alterado. Y ello pese a que la persistencia de la COVID-19 y la falta de perspectiva impiden ponderar el verdadero alcance y la naturaleza, coyuntural o estructural, de los cambios que aquí nos ocupan, siéndole aplicable a la pandemia "la cita apócrifa atribuida a un primer ministro chino, según la cual, bien avanzado ya el siglo XX, aún era pronto para hacer una valoración de la Revolución Francesa" (Roldan, 2020).

En cualquier caso, en este incierto escenario se abren paso algunas certezas. Por una parte, la universalidad del coronavirus ha precipitado o acelerado ciertas tendencias emergentes en relación a la desglobalización (Sulkoswski, 2020), la ausencia de liderazgos en la gobernanza mundial, el 
limitado funcionamiento del ordenamiento jurídico internacional y la fragilidad de la cooperación (Sander y Rudall, 2020) o la importancia de la seguridad humana (Tanaka, 2019), que apuntarían hacia un cambio de paradigma en la sociedad internacional. Y por la otra parte, la COVID-19 ha impactado de pleno en un aspecto determinante en todas estas transformaciones, como es la movilidad humana transfronteriza y, en particular, la libre circulación en el seno de la Unión Europea.

De hecho, la movilidad internacional de personas ha sido sin duda una de las señas de identidad de la globalización, junto al comercio mundial de bienes y servicios, las transferencias de capital, y la conectividad de la información, gracias a Internet y las redes sociales. La importancia de cada uno de estos cuatro vectores es fluctuante, habiéndose apreciado que en periodos de crisis los tres primeros se mantienen o se ralentizan, a la par que se dinamiza la globalización del conocimiento y la tecnología (Fanjul, 2019). El punto de inflexión estriba en que, a nuestro juicio, en ninguna de las crisis contemporáneas previas, ya fuera la crisis del petróleo en la década de los 70 del pasado siglo, las crisis económicas y financieras del siglo XXI, o la crisis de seguridad tras el $11-\mathrm{S}$, se ha producido una quiebra tan abrupta en la movilidad humana. Con todo, el impacto de este desplome no ha sido homogéneo, ya que el sentido y la intensidad de la movilidad humana varían según dónde pongamos el foco, destacando en términos cuantitativos y cualitativos la que acompaña a los procesos de integración regional, y muy especialmente al europeo (Malamud, 2011).

En este orden de cosas, de todos es sabido que la integración europea ha pivotado desde sus orígenes en torno a las llamadas cuatro libertades básicas: libre circulación de mercancías, de personas, de servicios y de capitales. También que desde 1992 la libre circulación de personas constituye la esencia del estatuto de la ciudadanía europea, un elemento determinante en la configuración de la actual UE. Sin perjuicio de que tanto la libre circulación de personas ad intra como la migración proveniente de terceros países hacia la UE han suscitado las críticas de populistas y euroescépticos, que han hecho de ellas un hilo conductor de sus respectivas narrativas (Consejo Vasco del Movimiento Europeo, 2018), su importancia para el proyecto europeo ha sido indiscutible hasta la eclosión de la COVID-19. Pero, desde el momento en que la UE se convirtió en epicentro de la pandemia, la libre circulación de personas ha pasado de ser pilar de la integración a convertirse en el principal obstáculo para la lucha contra la enfermedad. La limitación, a priori temporal, de este derecho por razones de orden público, salud y seguridad pública se convierte en la medida por excelencia para impedir la expansión acelerada de los contagios, evitando así el colapso de los sistemas de atención sanitaria. Como tendremos ocasión de comprobar, en el marco de la UE se han adoptado 
un conjunto de medidas legislativas y operacionales, encaminadas al cierre tanto de las fronteras interiores como de las fronteras comunes exteriores para impedir la entrada de extranjeros, además de las cuarentenas, el autoaislamiento y los confinamientos obligatorios decretados por la mayoría de los gobiernos de los 27 Estados miembros. Simultáneamente, las Instituciones Europeas, con la Comisión a la cabeza, han intentado que estas restricciones a la libre circulación de personas se aprobaran e implementaran de forma coordinada entre todos los socios europeos, a fin de evitar el caos económico y garantizar la vigencia de las otras tres libertades básicas, que son vitales para mantener en funcionamiento el mercado interior y los servicios que la gestión de la crisis de salud pública requiere.

La premisa de partida del presente trabajo es que, a pesar de que las actuales limitaciones a la libre circulación de personas son a priori temporales, al estar vinculadas al nacimiento y persistencia de la enfermedad, podrían sin embargo haber llegado para quedarse. La inadecuación del actual marco jurídico regulador de la libre circulación para afrontar una pandemia de dimensiones y duración desconocida, sumado a la quiebra del sistema Schengen, habría provocado en primera instancia una modificación fáctica del contenido y alcance de este derecho que, de confirmarse, podrían condicionar el desarrollo futuro de la UE a medio y largo plazo. En este sentido, cabe recordar que la pandemia ha coincidido en términos cronológicos con la apertura de la Conferencia sobre el futuro de Europa, cuyo inicio estaba previsto para el pasado 10 de mayo, fecha en la que se conmemoró el $70^{\circ}$ aniversario de la Declaración Schuman. Habida cuenta del desarrollo de la pandemia, entre las cuestiones que la realidad europea exige abordar, figura el debate sobre en qué medida sigue siendo válidos tanto la base jurídica en el derecho primario y derivado de la libre circulación de personas, como la normativa que regula el cierre y reapertura de las fronteras interiores y exteriores por razón de orden público, salud y seguridad. Por consiguiente, dicha Conferencia debe comenzar cuanto antes y hacerlo con renovado brío, incluyendo el debate sobre si en la nueva UE que se está pergeñando la libre circulación sigue siendo la regla y los límites la excepción o, por el contrario, se ha materializado una inversión definitiva mediante restricciones consolidadas a la libre circulación basada en la unilateralidad, en detrimento de los sistemas de coordinación e integración comunitaria sobre los que Schengen se sustenta.

Así lo atestigua el considerando B de la Resolución del Parlamento Europeo, de 18 de junio de 2020, sobre la posición del Parlamento Europeo sobre la Conferencia sobre el Futuro de Europa (2020/2657(RSP):

la crisis actual de la COVID-19 ha demostrado, a un coste muy alto, que la Unión sigue siendo un proyecto inacabado, y que la Conferencia debe abordar de mejor forma la incapacidad para garantizar la solidaridad y la coordinación, 
así como los choques económicos, sanitarios y sociales, y los ataques en curso contra los derechos fundamentales y el Estado de Derecho.

Para corroborar esta hipótesis, aunque en el momento de conclusión del presente trabajo la libre circulación de personas y el control de las fronteras tanto interiores como exteriores en la UE sigue siendo objeto de continuos retrocesos y avances, que se suceden sin solución de continuidad al ritmo que marca la evolución de una pandemia que parece lejos de darse por concluida, hemos adoptado una estructura basada en una división en tres fases: antes de la pandemia; en la pandemia, incluyendo la primera ola y los rebrotes en curso; y la post pandemia, cuyo horizonte se vislumbra plausible, al estar vinculado a la obtención de una vacuna eficaz, lo que presumible tendrá lugar dentro de los próximos dos años, que es justamente el periodo de tiempo durante el cual está previsto que se desarrolle y culmine la antedicha Conferencia sobre el futuro de la UE. Por ello, tras estas consideraciones preliminares (I), en el siguiente epígrafe nos situaremos en la fase previa a la pandemia para examinar brevemente, a modo de simple recordatorio, el marco jurídico normativo regulador de las limitaciones a la libre circulación de personas en el Derecho Internacional Público y en el Derecho de la UE (II). A renglón seguido abordaremos las restricciones a la libre circulación que se adoptaron como respuesta a la crisis sanitaria (III), así como el intento de restablecimiento del statu quo, y la dinámica de retrocesos y avances tras los rebrotes o segundas oleadas (IV). Finalmente, formularemos algunas reflexiones en relación al alcance que el rediseño de la libre circulación puede tener en el estatuto de ciudadanía y en el futuro modelo de la UE que se está dilucidando (V).

Un apunte final. Esta contribución da continuidad a una línea de investigación consolidada, en la que se insertan diferentes publicaciones previas sobre la materia. En particular, destaca un artículo titulado "Límites a la libre circulación de personas por razón de orden público, seguridad y salud pública: una revaluación a la luz de la jurisprudencia del TJUE", publicado en 2014 en la Revista de Derecho Comunitario Europeo, que incide de pleno en la hipótesis que aquí desarrollamos y nos proporciona el bagaje previo imprescindible para tener una visión panorámica de la materia. Conocer el funcionamiento y los problemas que con anterioridad presentaba la aplicación de los restricciones a la libre circulación por razones de orden público, salud o seguridad pública nos permitirá identificar con precisión los desafíos extra que ha traído consigo la pandemia, y, sobre todo, reflexionar si las la dinámica de restricciones y restitución de la movilidad humana y del control de fronteras en la que nos encontramos actualmente inmersos es coyuntural, o por el contrario marca un punto de inflexión que supondría la reconfiguración o incluso la derogación de un derecho clave en el proyecto de integración, con las consecuencias que eso comportaría para el futuro de Europa.

Araucaria. Revista Iberoamericana de Filosofia, Politica, Humanidades y Relaciones Internacionales, año $22, \mathrm{n}^{\circ} 45$. Tercer cuatrimestre de 2020. Pp. 311-335. ISSN 1575-6823 e-ISSN 2340-2199 https://dx.doi.org/10.12795/araucaria.2020.i45.13 


\section{A modo de recordatorio: marco jurídico normativo internacional y europeo del derecho a la libre circulación de personas}

Tanto la adopción de medidas restrictivas de la libre circulación como la reactivación segura y efectiva de la movilidad internacional exige que el abanico de posibles medidas y recomendaciones estén bien fundadas en el marco jurídico normativo internacional y europeo relativo a la libre circulación de personas, que dentro de esta categoría incluye a los trabajadores.

Para ello, es necesario precisar dicho marco brevemente, recordando el entramado de tratados multilaterales que están vigentes para todos los Estados parte, entre los que se incluyen los 27 miembros actuales de la UE, y cuya obligatoriedad no ha resultado afectada, por tres razones. Primera, porque dichos tratados pueden contribuir a la recuperación de la movilidad internacional. Segunda, porque también pueden servir de base para cuestionar las medidas restrictivas de la movilidad mantenidas o adoptadas ex novo en perjuicio de los intereses y del derecho de la UE. Y tercero, porque constituyen el derecho aplicable ante el previsible aumento de la litigiosidad ad futurum, en forma de demandas interestatales, o de personas físicas y jurídicas frente a los Estados miembros, ya sea por la adopción de restricciones a la libre circulación y/o por su mantenimiento indebido.

En el plano universal, dicho marco estaría compuesto por dos pilares. Por una parte, el artículo (art.) 13 de la Declaración Universal de Derechos Humanos de 10 de diciembre de 1948, que consagra la libre circulación interna de los nacionales para entrar, circular y salir del propio país, pero permite que todos los derechos sean limitados en tiempos de emergencia por razones de orden público. Y por la otra, el art. 12 del Pacto Internacional de Derechos Civiles y Políticos de 16 de diciembre de 1966, que reconoce que tanto la libre circulación interna (entrada, salida y movilidad) como la libre circulación internacional, podrán ser objeto de restricciones previstas en la ley necesarias y proporcionales para proteger la seguridad nacional, el orden público, la salud o los derechos y libertades de terceros. El control no jurisdiccional de las mismas compete al Comité de Derechos Humanos ONU, en cuya Observación General $\mathrm{n}^{\circ} 27$ de 1999 relativa a la libre circulación, se afirma que la proporcionalidad debe respetarse no sólo en la ley que defina dichas limitaciones, sino también por las autoridades administrativas y judiciales que la apliquen. En esta misma línea, en la Directrices ONU relativas a la COVID-19 se reitera que las medidas restrictivas de la movilidad humana no pueden ser discriminatorias por ninguna razón, incluyendo la nacionalidad.

Por lo que se refiere al ámbito regional europeo, la libre circulación se sustenta tanto en el marco del Consejo de Europa como en el de la UE. En el primero, desataca el art. 2 del Protocolo $n^{\circ} 4$ al Convenio Europeo de Derechos 
Humanos y Libertades Fundamentales (CEDHLF) de 16 de septiembre de 1962, donde se estipula que toda persona que se encuentra legalmente en el Estado tiene derecho a entrar, circular y salir, derechos que puede ser limitados legalmente por razones de seguridad nacional, orden público, prevención del delito, protección de la salud y derechos y libertades de terceros. Dichas restricciones habrán de ser legales, necesarias y proporcionales, y podrán aplicarse solo en ciertas zonas determinadas. A sensu contrario, el CEDH avalaría la adaptación de medidas de reactivación de la actividad económica también por zonas, que bien pudieran ser transfronterizas. El control jurisdiccional de todas las medidas, tanto las limitativas como las reactivadoras, corresponde al TEDH que, al ponderar su legalidad, finalidad, necesariedad y proporcionalidad, reconocerá un mayor margen de apreciación estatal cuanto mayor sea el consenso europeo al respecto.

Por su parte, el marco jurídico normativo de la libre circulación en la UE lo conforman en el derecho primario los arts. 20, 21.1, 26, 45.3 y 52.1 y 62 del TFUE, así como 45 y 52.2 de la Carta de Derechos Fundamentales de la UE. Y en el derecho derivado, la Directiva 2004/38/CE 29 de abril de 2004, transpuesta en España mediante Reales Decretos 240/2007 y 987/2015. La libre circulación está sometida a limitaciones por razón de orden público, salud y seguridad pública adoptadas por las autoridades nacionales conforme a sus necesidades, que no son necesariamente comunes, y que suelen variar con el tiempo, al igual que las circunstancias de su levantamiento. Las medidas limitativas han de cumplir con una serie de garantías normativas sustantivas (necesariedad, proporcionalidad y no discriminación) y procedimentales, sometidas al control crítico y continuado que en cada caso ejerza el TJUE. En todas las crisis previas, y la pandemia sanitaria no tiene porqué ser una excepción, el TJUE ha afirmado que las autoridades nacionales están obligadas a respetar el derecho a la tutela judicial efectiva de los ciudadanos de la Unión o sus familiares afectados por las medidas restrictivas de la libre circulación, que les faculta a impugnar la procedencia de tales medidas mediante a interposición de verdaderos recursos judiciales tanto nacionales como en el plano de la UE.

Finalmente, interesa destacar que, si los Estados miembros deciden tanto restringir como restituir la libre circulación, no podrán discriminar por razón de nacionalidad a los ciudadanos europeos, ni tampoco invocar la salud pública para imponer restricciones selectivas a los extranjeros con fines económicos. A diferencia del orden y la seguridad pública, cuya definición común en el ámbito europeo es tentativa o emergente, la salud pública sí presenta un contenido y perfiles objetivables en la UE, identificándose con el riesgo sanitario de enfermedades epidémicas decretadas como tales por la OMS.

Una vez expuesto someramente el entramado de normas internacionales y europeas vigentes y como se han interpretado y aplicado hasta la llegada de 
la COVID-19, pasaremos a analizar los nuevos desarrollos restrictivos que la pandemia ha supuesto en la libre circulación y, por añadidura, en el control de fronteras en la UE.

\section{Las medidas restrictivas de la libre circulación de personas en la ue durante el origen y la expansión de la pandemia}

La cronología, expansión y situación actual de la pandemia en la UE sobrepasa los límites de la presente contribución, por lo que nos detendremos solo en aquellos datos que consideramos relevantes para nuestro objeto de estudio.

Así, conviene recordar sucintamente que la OMS reportó la existencia de los primeros brotes de la enfermedad bautizada como COVID-19 en China en diciembre de 2019. Un mes después, el 30 de enero de 2020, ante el aumento de contagios y la mortalidad, el Comité de Emergencia de esta organización, siguiendo sus propios protocolos, declaró formalmente la existencia de una Emergencia de Salud Pública de Importancia Internacional (ESPII o PHEIC en sus siglas inglesas. Esta declaración vino acompañada de una serie de recomendaciones a la comunidad internacional para frenar el ritmo de difusión de la enfermedad, si bien a día de hoy resulta llamativo que entre ellas inicialmente no estaban previstas las restricciones a los viajes o al comercio, puesto que la información disponible en aquel momento hacia dudar de la eficacia de tales medidas en la lucha contra la enfermedad. Apenas un mes más tarde se conoció la existencia de los primeros casos en algunos miembros de la UE, si bien fue en el norte de Italia, la zona cero de la enfermedad en Europa, donde desde finales de febrero se venía registrando un aumento preocupante de casos. Dichos casos resultaron ser la punta de un iceberg, pues pocos días después la totalidad de los 27 socios reconocieron tener infectados por la COVID-19. De hecho, el Centro Europeo para el Control y Prevención de Enfermedades (ECDC en sus siglas inglesas) emitió un informe con fecha 2 de marzo de 2020 alertando de la expansión generalizada de casos y focos de infección en la práctica totalidad del territorio de la UE. El 11 de marzo de 2020 la OMS declaró formalmente que el brote de la COVID-19 había alcanzado la consideración de pandemia lo que, desde el punto de vista del Derecho Internacional, no implicó sin embargo consecuencias ni obligaciones jurídicas distintas o añadidas para los Estados parte en esta organización, pero sí tuvo importantes efectos para la UE, tanto en su dimensión de organización de integración como a nivel de sus Estados miembros.

Cronológicamente se ha constatado que dicha declaración habilitó a los gobiernos de los Estados miembros de la UE para recurrir al llamado 
genéricamente "derecho de excepción" que, ante una situación extraordinaria de crisis grave, permite excluir temporalmente la normalidad constitucional y limitar, restringir o suspender los derechos fundamentales, en especial el derecho a la libre circulación. Desde la óptica comparada, este derecho de excepción se traduce constitucionalmente en diferentes figuras, que según la carta magna en cuestión puede llamarse estado de alarma, excepción, sitio, o incluso estado de guerra (Fernández Rodríguez, 2020). Como atestigua un Informe del Parlamento Europeo, durante la primera quincena de marzo en 7 Estados miembros partiendo de marcos constitucionales similares se adoptaron diferentes estatutos, que tenían como denominador común restricciones parciales o totales de la libre circulación dentro de sus territorios y el cierre de las fronteras. En el caso de España, en virtud del artículo 116 de la Constitución, desarrollado por la Ley Orgánica 4/1981, de 1 de junio, el gobierno optó, no sin polémica, por decretar el estado de alarma mediante el RD 463/2020, de 14 de marzo. El objetivo primordial, aunque no único, del citado primer Real Decreto y de las cuatro prórrogas posteriores de las que ha sido objeto ${ }^{2}$, es el de prohibir con carácter general la movilidad humana ad intra y ad extra, salvo excepciones tasadas, lo que para un sector de la doctrina constitucionalista española es ir mucho más allá de lo que la normativa permitía (Fernández de Gatta Sánchez, 2020).

De hecho, la pandemia ha sido un test de estrés para los pilares europeos de la libre circulación y el control de fronteras desde tres perspectivas complementarias (Montalvo, 2020), ya que a las referidas restricciones de la movilidad decididas por cada Estado en relación a su propio territorio, hay que añadir la problemática de la reintroducción, también unilateral, de las fronteras interiores dentro del área Schengen, y del cierre de las fronteras exteriores de la Unión.

\subsection{La inacción de la UE en relación a derecho a la libre circulación}

Ya ha quedado de manifestó que tanto el derecho primario como el secundario de la UE admiten las restricciones clásicas por razones de orden público, seguridad y salud pública aplicables a la libre circulación de personas. La novedad estriba en que estas restricciones, tal y como hasta ahora habían sido invocadas, aplicadas y controladas jurisdiccionalmente, no se ajustan a las exigencias impuestas por la pandemia, que ha desequilibrado por completo el ya de por si delicado binomio salud versus libre circulación. De hecho, la respuesta de la UE como organización supranacional a este reto ha sido tan dificultosa

\footnotetext{
${ }^{2}$ El Real Decreto 463/2020 se prorrogó por primera vez el 27 de marzo (hasta el 12 de abril), por segunda vez el 10 de abril (hasta el 26 de abril), por tercera vez el 24 de abril (hasta el 9 de mayo) y por cuarta vez el 6 de mayo (hasta el 24 de mayo).
}

Araucaria. Revista Iberoamericana de Filosofia, Política, Humanidades y Relaciones Internacionales, año $22, \mathrm{n}^{\circ} 45$. Tercer cuatrimestre de 2020. Pp. 311-335. ISSN 1575-6823 e-ISSN 2340-2199 https://dx.doi.org/10.12795/araucaria.2020.i45.13 
como la que inicialmente dieron los Estados miembros, pero mucho más lenta, al partir de unas premisas determinadas, que constreñían extraordinariamente tanto su capacidad de respuesta como el alcance de la misma.

En primer lugar, hay que reseñar lo que pudiéramos denominar condicionantes jurídicos estructurales, que tienen que ver con el hecho de que, por una parte, conforme al art. 4 del TUE, la salvaguarda de la seguridad nacional sigue siendo "responsabilidad exclusiva de cada Estado miembro". Y, de otra parte, que las competencias de la UE en materia sanitaria son de apoyo, coordinación y complemento de las políticas y acciones de los Estados miembros (arts. 4 y 6 del TFUE), y están sometidas en su aplicación a los principios de subsidiariedad y proporcionalidad. La Comisión no dispone pues de iniciativa legislativa, y tampoco de recursos propios ni en el ámbito de la seguridad ni en el de la salud pública, donde el desarrollo operacional más relevante fue la creación en 2005 del ya aludido Centro Europeo para el control y la prevención de enfermedades (ECDC). No obstante, además de sus limitaciones competenciales, la Comisión se ha mostrado muy reticente a explorar nuevas vías que le hubieran permitido adoptar normas en materia sanitaria para corregir o complementar las preexistentes en materia de libre circulación. Así se desprende de la respuesta escrita a la pregunta que le formularon tres eurodiputados españoles acercar del posible recurso al art. 168.5 del TFUE como base jurídica habilitante para presentar una propuesta de reglamento en la lucha contra la pandemia, dado que, en opinión de los requirentes, los Estados miembros carecen por sí solos de la capacidad necesaria para responder con eficacia. La Comisión contestó recordando que estaba "colaborando" con las autoridades nacionales para proteger a los ciudadanos, a la par que intentando mantener el flujo de mercancías para, a continuación, evitando pronunciarse sobre el objeto de la pregunta, afirmar que sus propuestas de mejora de la gestión de la crisis consistían en reforzar la Agencia Europea de Medicamentos y asignar un papel más destacado al ECDC. Este centro, al ser preguntado sobre las medidas a adoptar para controlar la propagación de la enfermedad, no ha dudado en afirmar que "corresponde a cada Estado miembro tomar decisiones de acuerdo con las circunstancias locales y es necesario sopesar muchos factores al tomar esa decisión. Por lo tanto, no es posible, ni la función de la ECDC, hacer ningún comentario sobre las decisiones específicas de los Estados miembros ${ }^{3}$ ".

En segundo lugar, a estos condicionantes competenciales se suma el hecho de que la UE había previsto, pero no prevenido, la posibilidad de una pandemia de esta envergadura (Sanahuja, 2020: 31), y ni remotamente anticipó sus efectos sobre el proceso de integración. Y, finalmente, pero no menos importante, la ${ }_{3}$ Https://maldita.es/malditaciencia/2020/03/16/coronavirus-agencia-europea-aglomeraciones-
cierre-escuelas/ 
eclosión de la COVID19 se produjo en un momento especialmente delicado para la UE, instalada en una crisis sistémica, sobre todo de solidaridad, y justo cuando se estaba perfilando la Conferencia para el futuro de Europa cuyo objetivo es fijar las prioridades de una renovada UE a medio y largo plazo y acometer las reformas institucionales y de los Tratados constitutivos necesarias para ello.

El escenario hasta aquí expuesto sirve para comprender la falta de liderazgo de la UE en la coordinación, gestión y control de las medias restrictivas de la libre circulación de personas, punta de lanza de la lucha contra la propagación de la enfermedad. De hecho, por las razones ya expuestas de tipo jurídico competencial, la UE ha dejado por completo en manos de los Estados miembros la vigencia efectiva de este derecho clave de la ciudadanía europea, puntal del proceso de integración, renunciando de facto a controlar si se han cumplido o no las garantías normativas y procesales existentes, e ignorando la consolidada jurisprudencia previa del TJUE relativa el control judicial de dichas garantías. Constada su incapacidad y/o inoperancia, la respuesta de la UE se ha centrado en el infructuoso intento de coordinar las otras dos perspectivas del binomio salud pública-libre circulación, lo que hemos denominado la problemática de la reintroducción unilateral de los controles en las fronteras interiores dentro del área Schengen, y del cierre de las fronteras exteriores de la Unión.

\subsection{El restablecimiento de los controles en fronteras interiores y el cierre de las fronteras exteriores de la Unión: La (fallida) coordinación por la UE de las restricciones a la libre circulación}

En relación a la reintroducción unilateral de las fronteras interiores, la primera de las Instituciones en reaccionar fue la Comisión, publicando simultáneamente el 16 de marzo de 2020 dos documentos de soft-law. El primero era unas "Directrices sobre medidas de gestión de fronteras para proteger la salud y garantizar la disponibilidad de los bienes y de los servicios esenciales", en las que explicaba el giro copernicano que se disponía a dar, ya que solo cuatro días antes había criticado sin ambages las prohibiciones de viaje decretada por la administración Trump 5 . Y, el segundo se trataba de una Comunicación dirigida al PE, al Consejo Europeo y al Consejo titulada. "COVID-19: Restricción temporal de los viajes no esenciales a la UE", en las

\footnotetext{
${ }^{4}$ COVID-19 Directrices sobre medidas de gestión de fronteras para proteger la salud y garantizar la disponibilidad de los bienes y de los servicios esenciales (2020/C 86 I/01), C(2020) 1753 final. DOUE C 86/I/1, de 16 de marzo de 2020.

${ }^{5}$ Joint Statement by the President von der Leyen and President Michel on the U.S. travel ban of 12 March 2020, https://ec.europa.eu/commission/presscorner/detail/en/STATEMENT_20_449

${ }^{6}$ Comunicación de la Comisión al Parlamento Europeo, al Consejo Europeo y al Consejo. Covid 19: Restricción temporal de los viajes no esenciales a la UE, COM(2020) 115 final, de 16 de marzo de 2020 .
} 
que se detallan los objetivos, ámbito de aplicación y alcance de las restricciones a la movilidad transfronteriza de extranjeros hacía y en la UE, cuya adopción proponía.

Respecto al objetivo, la Comisión instó al Consejo Europeo a actuar rápidamente, para conseguir que todos los Estados parte en el sistema Schengen adoptasen una decisión coordinada "a fin de aplicar una restricción temporal de los viajes no esenciales de terceros países al espacio UE +". El ámbito de aplicación territorial es, como su propio nombre indica, el "Espacio UE+" que comprende todos los miembros de la UE, con la particularidad de que Irlanda, que lo es, y el Reino Unido que en esa fecha ya no lo era, podrían sumarse por la vía del opting-in, ya que comparten una zona de fronteras abiertas llamada "Área de viajes comunes" (CTA en sus siglas inglesas). El ámbito de aplicación personal de las restricciones afectaría a los nacionales de todos los terceros Estados, pero excluyendo a los británicos, quienes seguirán recibiendo el mismo trato que los ciudadanos de la UE hasta finales de 2020. Además, se establecían dos excepciones genéricas a dichas limitaciones. La primera, la de quienes siendo nacionales del Espacio UE+ y sus familiares, pretendieran regresar a su hogar, o la de aquellos extranjeros que tuvieran reconocido el estatuto de residentes de larga duración en la UE y quisieran también volver a su domicilio habitual. Y la segunda, la de los viajeros que, con independencia de su nacionalidad, tuvieran una función o necesidad esenciales tales como profesionales sanitarios, trabajadores transfronterizos, transportistas, diplomáticos, militares, pasajeros en tránsito o quienes viajasen por motivos familiares imperativos. Finalmente, el ámbito temporal de las restricciones no era indefinido, sino que se extendía a treinta días desde su aprobación, aunque estaban previstas las prórrogas que luego se materializaron en función de la evolución de la situación.

La lógica del discurso de la Comisión en relación a estas restricciones a la movilidad de personas dentro de la UE era sobre el papel impecable. Partiendo de que las fronteras exteriores de la UE deben servir de perímetro de seguridad para todos los Estados Schengen, las restricciones temporales a la movilidad son eficaces si se deciden y ejecutan en la totalidad de las fronteras exteriores simultánea y uniformemente. Por consiguiente, la Comisión concluye que, si el control de las fronteras exteriores se realiza de forma coordinada y al unísono, dejarían de estar justificado los controles en las fronteras interiores que con ocasión de la pandemia habían sido impuestas unilateralmente por cada Estado miembro. Así, al día siguiente el Consejo Europeo, en unas Conclusiones adoptadas tras su reunión extraordinaria del 17 de marzo, la primera de una serie celebrada por videoconferencia, endosó las propuestas de la Comisión, tanto la relativa a la restricción temporal de los viajes como a la Directrices sobre bienes esenciales. La novedad de ambos instrumentos, tanto el adoptado 
por la Comisión como el ratificado por el Consejo Europeo, radica en que ninguno de ellos se refiere ni invoca al Código de Fronteras Schengen de 2016 ni tampoco al resto del acervo Schengen, porque las Instituciones de la Unión eran conscientes de dos realidades normativas.

Primera, que el Código de fronteras no contempla el cierre general y simultaneo de las fronteras exteriores. Y segunda que, por el contrario, el régimen regulador de las fronteras interiores si prevé, como a continuación expondremos, hasta tres posibles escenarios en los que cabría restablecer los controles en las fronteras intra Schengen, pero ninguno de ellos de forma colectiva y permanente, a causa de una amenaza común a la salud pública, como era el caso. En esta tesitura, la UE ha asumido la inadecuación del acervo normativo regulador de la libre circulación y del control de fronteras para afrontar una situación inédita, que ha obligado a medidas excepcionalísimas, de reinstauraciones de esos controles internos que ya se habían puesto en marcha antes, pero nunca a causa de una pandemia, así como del cierre de las fronteras exteriores, acordado tras la mal llamada crisis de los refugiados sirios. Pero como se ha apuntado certeramente "del cierre simultáneo y colectivo de fronteras interiores y exteriores, no hay antecedentes" (Donaire Villa, 2020).

En efecto, el capítulo II (arts. 25 al 30) del Código Schengen contempla el restablecimiento temporal de los controles en las fronteras interiores, pero solo unilateralmente, es decir, cuando un Estado miembro detecte una amenaza grave de orden público o para su seguridad interior. Hasta el presente esas amenazas respondían a fenómenos conocidos, como el terrorismo internacional o los flujos masivos de migrantes, pero nunca de naturaleza sanitaria. Es por ello que el "Marco general del Código Schengen para el restablecimiento temporal de controles fronterizos en las fronteras interiores" (art. 25) resulta inadecuado, ya que parte de unos presupuestos a los que la COVID-19 no obedece, tales como que dichas medidas restrictivas sean el último recurso, lo que significa que puedan existir medios previos alternativos, que se adopten por periodos de 30 días renovables sin superar los seis meses, o que sean objeto de preaviso y se les notifique a los demás Estados miembros con "al menos cuatro semanas de antelación" (art. 27.1). Condiciones y cálculos todos ellos factibles en las amenazas clásicas antes referidas, pero que de nada sirven frente a esta pandemia. Tampoco serían fácilmente exigibles, por la gravedad y el coste de vidas humanas, los criterios de proporcionalidad de las medidas en relación con la amenaza, ni el llamamiento para que el Estado en cuestión tenga en cuenta las "repercusiones probables del restablecimiento de los controles en la libre circulación de las personas dentro del espacio sin controles en las fronteras interiores" (art.26.b).

\footnotetext{
${ }^{7}$ Reglamento (UE) 2016/399 del Parlamento Europeo y del Consejo, de 9 de marzo de 2016, por el que se establece un Código de normas de la Unión para el cruce de personas por las fronteras (Código de fronteras Schengen), DOUE L 77, de 23 de marzo de 2016.
} 
A mayor abundamiento, tampoco podría aplicarse el segundo procedimiento que el mismo Código Schengen dispone para los casos excepcionales que requieren una actuación inmediata (art. 28), y que contempla el restablecimiento unilateral por cada Estado de los controles en sus fronteras durante 10 días, prorrogables en períodos que no sobrepasen 20 días y con un máximo de dos meses.

Finalmente, el precepto que, sin ser del todo idóneo, podría servir para abordar el cierre de fronteras interiores en el espacio Schengen en el caso de la actual pandemia, era el previsto en el art. 29, que ya hemos anticipado se invocó en 2015 durante la crisis de los refugiados sirios. A diferencia de los dos anteriores, presupone un fallo sistémico en forma de "deficiencias graves persistentes en los controles de las fronteras exteriores", en cuya virtud se permite que el Consejo, previa propuesta de la Comisión, recomiende a uno o varios Estados parte restablecer los controles fronterizos en todas sus fronteras interiores o en partes concretas de ellas. Este procedimiento colectivo y coordinado, a diferencia de los anteriores, no se basa en decisiones unilaterales, y faculta a los Estados que estén en esa situación excepcional, a alargar la duración de los controles en la frontera durante periodos continuados de 6 meses, prorrogables sucesivamente hasta llegar a un máximo de 2 años (art. 29.2).

Sin embargo, a la hora de afrontar la restitución de los controles fronterizos internos y externos, los Estados parte de Schengen han repetido los errores del pasado y, en lugar de optar por un enfoque coordinado y coherente, se han decantado por un mosaico de decisiones unilaterales, teóricamente avaladas por el segundo procedimiento (art. 28). Pero, a nuestro juicio lo han hecho forzando tanto el tenor literal como el espíritu del Código de Fronteras por dos razones tan evidentes como relevantes.

Primera, porque, aunque parece haberse dado por sentado, "Border Controls do not mean Border Closures" (Thym, 2020). Es decir, lo que Schengen permite ahora, como lo hizo en 2015, es el restablecimiento de los controles fronterizos, pero no el cierre a cal y canto de las fronteras y la derogación en bloque del derecho de libre circulación de los ciudadanos europeos mediante las prohibiciones de viaje, que es lo que de facto ha sucedido. Y, segundo, porque salvo un reducido grupo de Estados, entre los que se cuenta España, Francia e Italia, que establecieron restricciones totales a la movilidad interior, pero mantuvieron parcialmente sus fronteras abiertas, el resto de los socios Schengen optaron justo por el modelo contrario, que suponía un confinamiento interno más relajado, pero un cerrojazo total de las fronteras intra Schengen en flagrante violación del derecho vigente. Ambas circunstancias explicarían que, al adoptar las dos Comunicaciones examinadas la Comisión, omitiese por completo referirse o invocar el acervo Schengen. A ello se le suma que 
la temporalidad intrínseca a la reintroducción de controles en las fronteras interiores ha desaparecido por completo en la praxis, puesto que desde la eclosión de la pandemia la mayoría de los Estados, una vez notificado el cierre inicial de sus fronteras, lo han mantenido sine die, sin respetar las preceptivas solicitudes de prórroga ni los tiempos máximos previstos.

Todos estos indicios nos llevan a anticipar una reflexión que desarrollaremos en el apartado final de conclusiones: que la pandemia habría cristalizado, a modo de deja vù, un temor previamente diagnosticado, consistente en la quiebra, o peligro de muerte anunciada del espacio Schengen, debido al cuestionamiento de su filosofía y la inversión de sus principios. Basta recordar que antes de la pandemia la conjunción de factores adversos, entre los que destaca el terrorismo y la crisis de los refugiados, habría provocado que un representativo grupo de Estados parte (Alemania, Austria, Suecia, Dinamarca, y Noruega), con la aquiescencia de la Comisión, prorrogaran los controles en las fronteras intra Schengen más allá de lo permitido, convirtiendo en regla general lo que en el Código era una excepción y viceversa (De Somer, 2019). Resta indagar en qué medida con ocasión de la COVID-19 esta práctica recurrente de los Estados $\mathrm{y}$, sobre todo, la pasividad de la Unión para cumplir con su compromiso de garantizar la ausencia de controles y la libre circulación de personas, han hecho de Schengen una víctima más del coronavirus y, por ende, hasta qué punto esto supondría el principio del fin de la UE en su actual configuración.

En todo caso, es conveniente poner de manifiesto que ninguno de los tres procedimientos previstos en el Código de Fronteras autoriza a la Comisión a prohibir que los Estados parte puedan restablecer unilateralmente los controles fronterizos, ya que, como hemos insistido, son competentes en materia de salud pública y ejercen sus responsabilidades en el mantenimiento del orden público y la salvaguardia de la seguridad interior (art. 72 del TFUE). Pero lo que sí puede es llevar a cabo algo que hasta ahora ha hecho solo a medias. De una parte, supervisar si el restablecimiento de los controles en las fronteras, tanto interiores como exteriores, respeta los procedimientos y plazos exigidos, verificando que sean temporales, proporcionales y no discriminatorios entre nacionales y ciudadanos del resto de la UE. Y, de la otra, asegurarse, en su condición de guardiana de los Tratados, que las limitaciones a la libre circulación de personas por razones de salud pública cumplen con las garantías normativas y procesales previstas en el derecho primario y secundario, así como su eventual control jurisdiccional. Se trata, ni más ni menos, de que al albur de la pandemia no se socave el sistema de controles y contrapesos que rigen en la UE.

De hecho, del control político de las medidas restrictivas de la movilidad se encarga el Parlamento Europeo (PE), en especial a través de la Comisión de libertades civiles, justicia y asuntos de interior (LIBE), con marcado acento 
español ${ }^{8}$. En un primer informe fechado en 2016 con ocasión de la crisis de los refugiados, puso de relieve el elevado coste que, tanto en términos políticos como sobre todo económicos, supuso la inobservancia continuada del Código Schengen, y el consiguiente peligro de desaparición de este acervo. A esta denuncia se han sumados otros dos informes consecutivos de 2018 y 2019 , incidiendo en el incumplimiento que implica la prolongación indebida por los Estados parte de los controles en las fronteras interiores ${ }^{9}$. Afortunadamente, con ocasión de las restricciones acordadas durante la pandemia el PE sigue sin bajar la guardia, como atestiguan alguna de las preguntas dirigidas al Consejo, en el que se le requiere su opinión sobre si se ha respetado el acervo de la UE sobre libre circulación y control de fronteras ${ }^{10}$. En la misma línea, destaca la Resolución de 19 de junio de 2020, en la que el PE expresa su preocupación por el hecho de que aún subsistan los controles fronterizos intra Schengen y porque, a su juicio, la Comisión no está siendo lo suficientemente incisiva a la hora de liderar el retorno a un espacio Schengen plenamente operativo, "que reviste la máxima importancia de cara a salvaguardar el principio de libre circulación como uno de los principales logros de la integración europea, y como un requisito previo clave para la recuperación económica de la Unión tras la pandemia de COVID-19"11.

No obstante, cabe recordar que justo unos días antes, el 30 de marzo, la Comisión aprobó un paquete de dos comunicaciones en esta dirección. Una de ellas las "Directrices relativas al ejercicio de la libre circulación de los trabajadores"12, se emitieron con el propósito de garantizar un enfoque coordinado a escala de la UE en relación a los trabajadores que necesitaran cruzar las fronteras para llegar a su lugar de trabajo, al ejercer ocupaciones críticas o realizar actividades relacionadas con los servicios esenciales. La segunda, las "Directrices sobre la aplicación de la restricción temporal de los viajes no esenciales a la UE, sobre la facilitación del régimen de tránsito para la repatriación de los ciudadanos de la UE y sobre sus efectos en la política de visado ${ }^{13}$ ", son consecuencia de la asunción desde esas fechas por

\footnotetext{
${ }^{8}$ Internal border controls in the Schengen Area: is Schengen crisis-proof? Directorate General for Internal Policies, disponible en: https:/www.europarl.europa.eu/RegData/etudes/STUD/2016/571356/ IPOL STU\%282016\%29571356 EN.pdf

9 El futuro del espacio Schengen: los acontecimientos y retos más recientes en el marco de gobernanza de Schengen desde 2016, Dirección general de políticas interiores departamento temático de derechos de los ciudadanos y asuntos constitucionales

${ }^{10}$ Pregunta con solicitud de respuesta oral O-000037/2020 al Consejo Artículo 136 del Reglamento interno formulada por Juan Fernando López Aguilar en nombre de la Comisión de Libertades Civiles, Justicia y Asuntos de Interior, 18 de mayo de 2020.

${ }_{11}$ Resolución del Parlamento Europeo, de 19 de junio de 2020, sobre la situación en el espacio Schengen tras el brote de COVID-19 (2020/2640(RSP), disponible en: ttps://www.europarl.europa. eu/doceo/document/TA-9-2020-0175_ES.pdf

12 Comunicación de la Comisión. Directrices relativas al ejercicio de la libre circulación de los trabajadores (2020/C 102 I/03), DOUE C-102 I./2, de 30 de marzo de 2019.

${ }^{13}$ Comunicación de la Comisión. Directrices sobre la aplicación de la restricción temporal de los
} 
la Agencia Europea de la Guardia de Fronteras y Costas (Frontex) del control y la denegación de la entrada de todos los nacionales de terceros países cuyos viajes no tuvieran la consideración de esenciales. Como última muestra de los esfuerzos de la Comisión en pos de la recuperación de la libre circulación de personas que, como veremos a continuación, han fructificado solo parcialmente, destaca su recopilación continuada de las notificaciones emitidas por los Estados miembros informando de la reintroducción temporal del control fronterizo en las fronteras interiores de conformidad con los arts. 25 a 20 del Código de fronteras Schengen ${ }^{14}$.

\section{Restablecimiento parcial de la libre circulación de personas y nuevas restricciones: avances y retrocesos}

La combinación de la incapacidad y la falta de voluntad de la UE para actuar de forma rápida y decidida, verificando que las restricciones a la libre circulación, fuera y dentro de su territorio, eran conformes al derecho vigente y tenían un carácter provisional, no solo fue criticada por el PE. También el Alto Representante (Borrell, 2020), algunos de los gobiernos de los Estados miembros y, sobre todo, la propia ciudadanía, expresaron su preocupación por la gestión inicial. De hecho, compartimos la idea de que una de las notas distintivas de la pandemia frentes a otras crisis previas como la de refugiados, en las que también se restringió la movilidad humana, ha sido el clamor ciudadano en favor de más Europa y no de menos (Morillas, 2020).

Por eso, a mediados del mes de marzo la UE empezó a reaccionar trabajando en favor de la recuperación de la movilidad humana. El 15 de abril de 2020, la presidenta de la Comisión y el presidente del Consejo Europeo publicaron una "Hoja de ruta común europea el para el levantamiento de las medidas de contención de la COVID-19", en la que manifestaba la necesidad de una vuelta progresiva a la normalidad, ya que las medidas extraordinarias de confinamiento ni podían ni debían mantenerse indefinidamente, enfatizando la necesidad de realizar una evaluación continua de su proporcionalidad, transitando hacia la nueva normalidad con una planificación coordinada y un calendario de fases, a fin de recuperar la libertad de circulación y el espacio interior sin fronteras. En particular, el levantamiento de las medidas de contención se guiaría por tres principios básicos: prioridad a la salud pública; coordinación entre los Estados,

viajes no esenciales a la UE, sobre la facilitación del régimen de tránsito para la repatriación de los ciudadanos de la UE y sobre sus efectos en la política de visado (2020/C 102 I/02), DOUE C-102 I./3, de 30 de marzo de 2019.

${ }^{14}$ Temporary Reintroduction of Border Control, European Commision, disponible en: https:// ec.europa.eu/home-affairs/what-we-do/policies/borders-and-visas/schengen/reintroduction-bordercontrol_en 
con la Comisión como nexo; y respeto y solidaridad mutua entre todos los socios. Por su parte, la reapertura de las fronteras exteriores y el acceso de residentes en terceros países se acometerían en una segunda fase, en función de la propagación del virus fuera de la UE y los peligros de reintroducción.

Menos de un mes más tarde, el 13 de mayo, la Comisión publicó una nueva Comunicación titulada "Por un enfoque gradual y coordinado de la restauración de la libertad de circulación y del levantamiento de los controles en las fronteras interiores - COVID-19"15. Tras constatar la mejoría de la situación sanitaria en la UE, y que sus medidas anteriores habían logrado en parte mantener en funcionamiento el mercado único, consideró llegado el momento de restablecer la libre circulación y la integridad del espacio Schengen. Y lo hizo invocando tres argumentos claros. Primero, que Schengen es uno de los mayores logros de la integración europea, presente y futura. Segundo, que acabar con las restricciones era fundamental para la recuperación de la economía. Y tercero, que las limitaciones a la libre circulación "dañan nuestra forma de vida europea en una Unión donde los ciudadanos pueden viajar libremente a través de las fronteras, como trabajadores, estudiantes, familiares o turistas".

Con este argumentario, la Comisión invitó a los Estados miembros a que iniciasen un proceso de reapertura gradual de la movilidad transfronteriza dentro de la Unión, en el bien entendido que la supresión de las restricciones nacionales, de conformidad con el principio de no discriminación, debería venir acompañado del progresivo restablecimiento de la libre circulación y del levantamiento de los controles en las fronteras exteriores estructurado en tres fases. Así, en primer lugar, se suprimirían las restricciones nacionales a la libre circulación bajo el principio de no discriminación, de modo que cuando se reanudasen los viajes dentro de su territorio, el Estado en cuestión debía permitir también los desplazamientos transfronterizos desde regiones o países de la UE con situaciones epidemiológicas similares, facilitando así el tránsito fluido por razones personales y familiares. La fase dos consistiría en el levantamiento de todas las restricciones y controles relacionados con la COVID-19 en las fronteras interiores de la UE. Finalmente, la coordinación para lograr estos objetivos se canalizaría a través del "Grupo de la COVID-19 - Asuntos de Interior” y del mecanismo de Respuesta Política Integrada a las Crisis, hasta llegar a restablecer la integridad del espacio Schengen y la libre circulación sin restricciones ni fronteras de las personas, los trabajadores, los bienes y los servicios en la UE.

Pese a la insistencia de la Comisión en la importancia de coordinar la vuelta a la libre circulación, habida cuenta del caos unilateral que caracterizó

${ }^{15}$ Comunicación de la Comisión. Por un enfoque gradual y coordinado de la restauración de la libertad de circulación y del levantamiento de los controles en las fronteras interiores - COVID-19, DOUE C 169/30, de 15 de mayo de 2020. 
la adopción de las medidas restrictivas en la fase anterior, el 4 de junio Italia y España dirigieron una carta conjunta a la Comisión. En la misiva insistían en que la Comisión liderase la reapertura de las fronteras intra Schengen a partir de "criterios epidemiológicos, comunes, claros y transparentes" definidos por el ECDC. En relación a las fronteras exteriores, abogaban por la apertura progresiva, monitorizada también por el ECDC en función de la incidencia de la COVID-19 en terceros países. A su juicio, del éxito de ambas dependería en gran medida la percepción de la ciudadanía europea sobre la eficacia de la Unión a la hora de afrontar esta crisis, y la resiliencia de los principios fundamentales de la UE y del espacio Schengen. En suma, se evidenciaba un temor más que razonable de que también en esta fase se mantuviera la tendencia unilateralista, amén de una proliferación de acuerdos bilaterales heterogéneos entre socios vecinos, que confundiría a los ciudadanos, propiciando situaciones discriminatorias prohibidas por el derecho de la $\mathrm{UE}^{16}$.

La reacción de la Comisión no se hizo esperar, de modo que el 11 de junio en una Comunicación con ocasión de la tercera evaluación de la aplicación de la restricción temporal de los viajes no esenciales a la UE ${ }^{17}$ fijó dos fechas claves, recomendando a los Estados miembros que suprimieran las restricciones intra Schengen el 15 de junio de 2020, mientras que el levantamiento de cierre temporal de las fronteras exteriores para los viajes no esenciales a la UE se fijó para el 30 de junio. En ambos casos, la problemática jurídica era similar a la explicada con ocasión de la imposición de las restricciones: la inexistencia de una base en el derecho primario o secundario para articular una acción colectiva coordinada, y el mero carácter recomendatorio de los actos de la Comisión.

En relación a las fronteras interiores, la mayoría de los Estados miembros con alguna excepción como España, habían adelantado ya su intención de restablecer la libre circulación intra Schengen en esas fechas mismas fechas. No obstante, como cabría esperar, la práctica fue muy desigual. En un extremo, se situaría el ejemplo de Suecia, cuya política disidente, basada en una hipotética inmunidad comunitaria controlada que resultó fallida, trajo consigo que el resto de los socios nórdicos le excluyeran de la reapertura conjunta de sus fronteras. En el otro extremo, la sincronía mostrada por Portugal y España. Después de tres meses de cierre de sus fronteras, el 1 de julio se celebró una ceremonia formal al máximo nivel para testimoniar la adhesión de la Península Ibérica a la libre circulación y a Schengen, como parte del legado presente y futuro de la UE (Lisa, 2020).

\footnotetext{
${ }^{16}$ Medidas de contención y desescalada adoptadas por los gobiernos europeos frente al coronavirus (enero-julio 2020), CIDOB, disponible en: https://www.cidob.org/biografias_lideres_politicos/ organismos/union_europea/covid_19_la_respuesta_de_europa_contra_la_pandemia

${ }_{17}$ Comunicación de la Comisión al Parlamento Europeo, al Consejo Europeo y al Consejo sobre la tercera evaluación de la aplicación de la restricción temporal de los viajes no esenciales a la UE, COM(2020) 399 final, de 11 de junio de 2020.
} 
Mayor complejidad ha supuesto la reapertura de las fronteras exteriores de la Unión, tanto por la existencia de diferentes sensibilidades sobre la forma de gestionar la gradualidad como, en especial, sobre la elaboración del listado de terceros países cuyos nacionales pueden entrar en la UE. Así en principio en la Comunicación de 11 de junio se pactó de común acuerdo entre los socios levantar la restricción a la entrada en la UE a partir del 1 de julio a una lista muy reducida de 6 países, todos ellos europeos. Con posterioridad, en la Recomendación de la (UE) 2020/912 del Consejo, de 30 de junio de 2020, la lista cambió, ampliándose a 11 países de todos los continentes. Desde entonces, con una periodicidad de 15 días se han venido adoptando sucesivas Recomendaciones para actualizar las altas y bajas en el listado. A título de ejemplo, con fecha 28 de agosto de 2020 eran 11 los países cuyos nacionales podían entrar en el territorio de la UE (Australia, Canadá, Georgia, Japón, Nueva Zelanda, Ruanda, Corea del Sur, Tailandia, Túnez, Uruguay, y China, esta última bajo reciprocidad).

Por consiguiente, de forma gradual y con no pocas dificultades, la libre circulación de personas empezó a restablecerse conforme el plan de la Comisión. Sin embargo, el cumplimiento de las previsiones normativas ha estado supeditado desde el inicio de la pandemia a la voluntad política de los Estados miembros, y, sobre todo, a la evolución de los contagios. Tal y como los epidemiólogos habían vaticinado, desde principios de agosto la curva ascendió, registrándose nuevos brotes, locales al principio, y más extendidos después, debidos, entre otros factores, a la reanudación de la movilidad humana. La respuesta de un grupo relevante de socios de la UE, repitiendo patrones unilaterales bien conocidos, está siendo la de mantener las restricciones a la libre circulación o dar marcha atrás en su reinstauración, lo que obligó a la Comisión a adoptar el 4 de septiembre otra Recomendación al Consejo para mantener la coordinación y asegurar el traslado de información a escala de toda la UE, y no en grupos por afinidades o proximidad geográfica (i.e Mediterráneos, Visegrado, nórdicos). La propuesta de la Comisión establece cuatro ámbitos clave en los que los Estados miembros deben colaborar más estrechamente, a saber: 1) criterios comunes y umbrales para los Estados miembros a la hora de decidir si se introducen restricciones a los viajes, basados en el número total de casos que reporte cada país, el número de pruebas realizadas y el porcentaje de resultados positivos; 2) una cartografía de criterios comunes utilizando un código común de colores, que van del verde al rojo según el grado de propagación, pasando por el gris si no se dispone de información o el número de pruebas es insuficiente. Nótese que esto último presupone que cuanto más transparente y riguroso sea un Estado al trasmitir la información, más restricciones pueden imponérsel; 3) un enfoque común en relación a las medidas a aplicar a los viajeros procedentes de zonas de alto riesgo, según el 
color asignado a cada zona de origen; 4) información clara y oportuna a los ciudadanos sobre cualquier restricción. Cada Estado debe proporcionar, una semana antes de su entrada en vigor, información detallada sobre las futuras restricciones a la libre circulación o el levantamiento de las restricciones de viaje, y la misma se publica, también semanalmente en una plataforma virtual ad hoc bautizada gráficamente como "Re-open EU".

En conclusión, tras constatar que la evolución de la pandemia ha generado, y sin duda seguirá haciéndolo en el futuro inmediato, una dinámica de avances y retrocesos constantes en el restablecimiento de la libre circulación, lo que la UE ha de evitar a toda costa es lo que el Comisario de Justicia ha denominado certeramente "la cacofonía generada por las normas nacionales". Por ello, en caso de que se mantengan o adopten nuevas medidas restrictivas, se han implementado unos criterios, comunes, accesible e inteligibles para todos los ciudadanos europeos, $\mathrm{y}$, sobre todo y lo más importante, no discriminatorios. Pero, como de solito, su eficacia pasa necesariamente por la voluntad política de los Estados miembros y, sobre todo, porque las Instituciones y la ciudadanía europea velen activamente por su cumplimiento. De ello depende el mantenimiento de nuestra forma de vida y probablemente también el futuro de la propia UE.

\section{Reflexiones finales}

Comenzamos estas páginas planteándonos en qué medida la pandemia ha contribuido a cristalizar ciertas tendencias previas, tanto en el plano internacional como en el marco europeo. En este sentido, parece haber ratificado que los desafíos globales provocan un efecto de retracción en los Estados que, al menos en un primer momento, buscan la respuesta en nacionalismos e unilateralismos de toda índole, incluido el nacionalismo de fronteras y el epidemiológico (Jerónimo, 2020). A ello se le suma que la COVID-19 ha vuelto a colocar a la UE frente al espejo en un momento especialmente delicado, con una Conferencia sobre el futuro de Europa en puertas, en la que se dilucidará el modelo de integración para las próximas décadas. Es imperativo extraer las muchas lecciones aprendidas y trasladarlas a este foro. Entre ellas, compartimos plenamente la tesis de que "la crisis del coronavirus es un nuevo ejemplo de que la libre circulación de personas no es compatible con una integración a medias" (Feás, 2020). Aún a sabiendas de que la historia demuestra que durante los episodios críticos graves la integración europea se frena en seco, e incluso se producen retrocesos como los aquí hemos expuesto, es importante preservar el legado jurídico que, en forma de acervo normativo, Schengen incluido, ha permitido la creación de un espacio común sin fronteras, en el que los ciudadanos europeos circulan libremente. 
Hasta el presente, contamos con un sólido marco jurídico internacional y de derecho de la UE regulador de las restricciones posibles a la libre circulación. A dicho marco con ocasión de la pandemia se le han sumado un conjunto de normas de soft law en forma de recomendaciones, directrices y hojas de ruta, que han tratado de coordinar la acción de los Estados miembros tanto en la imposición de las medidas restrictivas como en su levantamiento. A este respecto, queremos recodar dos consideraciones, expuestas en trabajos anteriores, pero que hoy cobran especial relevancia. Primera que, al igual que en otros desarrollos recesivos previos, en la era pos-COVID-19 el ejercicio de la libre circulación con las limitaciones legalmente previstas, interpretadas restrictivamente, resulta imprescindible para garantizar la libertad, la igualdad y la no discriminación en una UE sin fronteras interiores. De ahí la necesidad de proteger los logros conseguidos frente a posibles derivas restrictivas, evitando dar por sentado su irreversibilidad. Y segunda, que es clave el control jurisdiccional ejercido tanto por los tribunales nacionales como por el TJUE para preservar los derechos inherentes al estatuto de la ciudanía europea y evitar restricciones a la movilidad y cierres de fronteras discriminatorios. Por ello nos congratulamos de la advertencia en forma de carta con que la Comisión ha reaccionado a la notificación hecha el 1 de septiembre por el gobierno de Hungría permitiendo solo la entrada a su país de sus propios nacionales, en flagrante discriminación del resto de ciudadanos europeos. Consentir la caída definitiva de Schengen no solo implica herir de muerte a la ciudadanía europea, sino que es bien sabido comporta un daño económico directo irreparable que, hoy más que nunca, la UE no se puede permitir, ya que choca frontalmente con el plan de recuperación económica recién acordado (Donaire Villa, 2020).

Finalmente, la preservación de la libre circulación exige conjugar decisiones estructurales con medidas operativas. En relación a las primeras, si finalmente la Conferencia sobre el futuro de Europa se salda con una propuesta de reforma de los Tratados Constitutivos, entre las mismas debería tener cabida que la salud pública sea una competencia compartida, para facilitar que las medidas de seguridad restrictivas de la libre circulación obedezcan a criterios uniformes en los 27 socios. Y, por lo que concierne a las medidas operativas, creemos que la traslación de los Protocolos de Homologación sanitarios y de rastreo de la enfermedad al ámbito del Espacio de Libertad, Seguridad y Justicia pueden ser muy útiles. Así, a partir del principio de confianza y reconocimiento mutuo, y mediante el recurso a instrumentos normativos similares a las órdenes europeas, se podrían acordar que la declaración de zona seguras y la acreditación sanitaria en origen fuera aceptada automáticamente con carácter general por los restantes Estados. Paralelamente existiría otra lista de zonas no seguras en las que no funcionaría la automaticidad, sino la revisión por cada Estado de la seguridad sanitaria de la contraparte, mediante el estudio caso a caso. 
En el discurso de la crisis entendida como oportunidad, nunca antes hemos tenido otra igual para relanzar la integración europea. La tan socorrida metáfora de la bicicleta, según la cual en el proyecto europeo lo importante era pedalear, aunque no supiéramos en qué dirección, ha dejado de ser válida. La COVID-19 ha desvelado que, en 2020 como en 1950, los Estados siguen siendo incapaces por sí solos de mantener su seguridad, incluida la sanitaria o de proporcionar a sus ciudadanos los bienes y los derechos que les asisten, incluida la libre circulación. Parafraseando al General de Gaulle, en la era COVID-19, la UE puede hacer suya la frase de "o yo o el caos". 


\section{Referencias bibliográficas:}

Borrell, J. "The post-coronavirus world is alredy here. Policy Brif, European Council on Foreign Relations", disponible en: https://www.ecfr.eu/ publications/summary/the_post_coronavirus_world_is_already_here

Consejo Vasco del Movimiento Europeo (Bengoetxea, J (coord). (2018). Nuevas narrativas para Europa.¿ Qué Europa reconstruir tras 60 años de los Tratados de Roma?. Midac, SL.

De Somer, M, (2020). "Schengen and Internal Border Controls", En From Tampere 20 to Tampere 2.0: Towards a new European consensus on migration', De Bruyker P. et altri (eds), European Policy Centre, 119-131.

Donaire Villa, F.J. (2020) "Cierre ¿provisional? de las fronteras europeas", Observatorio de Derecho Público, disponible en: dpbarcelona.net/ schengen-ante-coronavirus-cierre-provisional-las-fronteras-europeas/

Fanjul, E. (2019). "Los cambios estructurales dela globalización”, Blog Real Instituto Elcano, disponible en: https://blog.realinstitutoelcano.org/loscambios-estructurales-de-la-globalizacion/

Fernández Rodríguez, J.J. (2020). “Cuestiones constitucionales sobre el estado de alarma en España y la pandemia del COVID-19”. Documento de Opinión IEEE, disponible en http://www.ieee.es/Galerias/fichero/docs opinion/2020/DIEEEO43_2020JOSFER_alarma.pdf

Fernández de Gatta Sánchez, D. (2020). "Los problemas de las medidas jurídicas contra el coronavirus: las dudas constitucionales sobre el Estado de Alarma y los excesos normativos", Diario La Ley (9641).

Feás, E. (2020). "Las lecciones para Europa del coronavirus", disponible en: $\quad$ http://www.realinstitutoelcano.org/wps/portal/rielcano es/ contenido?WCM_GLOBAL_CONTEXT=/elcano/elcano_es/zonas_es/ feas-las-lecciones-para-europa-del-coronavirus

Jerónimo, P. (2020). "COVID-19- Nationalism and its tolls on citizenship and mobility rights in the European Union", UNIO EU Law Journal, The Official Blog, disponible en: https://officialblogofunio.com/2020/04/20/ covid-19-nationalism-and-its-toll-on-citizenship-and-mobility-rights-inthe-european-union/

Lisa, P. (2020) "Fronteras y Schengen: la importancia de los buenos ejemplos", Blog Elcano, 10 de julio de 2020, disponible en:

Malamud, A. (2011). "Conceptos, teorías y debates sobre la integración regional". Norteamérica, 6(2), 219-249.

Martín Martínez, M. (2014). "Límites a la libre circulación de personas por razón de orden público, seguridad y salud pública: una revaluación a la luz de la jurisprudencia del TJUE", Revista de Derecho Comunitario Europeo (49), 767-804. 
Martín-Moreno, J.M. (2020), "La OMS en su encrucijada", ARI 65, disponible en: http://www.realinstitutoelcano.org/wps/portal/rielcano_es/ contenido?WCM_GLOBAL_CONTEXT=/elcano/elcano es/zonas_es/ ari65-2020-martinmoreno-oms-en-su-encrucijada

Montalvo, S. (2020). "The COVID-19 Emergency and the Reintroduction of Internal Border Controls in the Schengen Area: Never Let a Serious Crisis Go to Waste", European Papers vol. 5, 523-535, disponible en: http://www.europeanpapers. eu/en/system/files/pdf version/EP EF 2020 I 016 Stefano Montaldo.pdf

Morillas, P. (2020). "Lecciones de una crisis global: coronavirus, orden internacional y futuro de la Unión Europea", CIDOB Notes Internacionals, disponible en: https://www.cidob.org/ca/publicacions/series_de_publicacio/ notes_internacionals/n1_231/lecciones_de_una_crisis_global_coronavirus_ orden_internacional_y_el_futuro_de_la_ue

Ortega, A. (2020). The U.S.-China Race and the Fate of Transatlantic Relations, Center for Strategic and International Relations, disponible en: http:// csis-website-prod.s3.amazonaws.com/s3fs-public/publication/200113 USChinaTranstlanticRelations.pdf

Pons Rafols, X (2020). "La COVI-19, la salud global y el Derecho Internacional: una primera aproximación de carácter institucional", REEI (39), DOI: 10.17103/reei.39.06

Recchi, E., et altri, (2019). "Estimating Transnational Human Mobility on a Global Scale", EUI RSCAS Working Paper 2019/30. Fiesole: European University Institute.

Roldán Barbero, J. (2020), "Reflexiones de un internacionalista sobre la pandemia 2. El tiempo y la Covid1-19 ", Blog Aquiescencia, disponible en https://aquiescencia.net/2020/06/12/reflexiones-de-un-internacionalistasobre-la-pandemia-2-el-tiempo-y-la-covid-19-por-javier-roldan-barbero/

Sanahuja, J.A. (2020), "COVID-19: riesgo, pandemia y crisis de gobernanza global", [en Manuela Mesa, coord.: Riesgos globales y multilateralismo: el impacto de la COVID-19, Madrid, Fundación Cultura de Paz, Anuario 2019-2020, 31- 2020, disponible en: https://ceipaz.org/wp-content/ uploads/2020/05/0.2020-ANUARIO-COMPLETO.pdf

Sander, B. and Rudall, J. (eds) (2020). "COVID19 and International Law", Opinio Iuris Symposium, disponible en : http://opiniojuris.org/wp-content/ uploads/B.-Sander-J.-Rudall-eds-COVID-19-and-International-LawOpinio-Juris-Symposium-copy-1.pdf

Sułkowski, Ł. (2020). "Covid-19 pandemic; recession, virtual revolution leading to de-globalization?". Journal of Intercultural Management, 12(1), 1-11.

Tanaka, A. (2019). "Toward a theory of human security". In Human Security and Cross-Border Cooperation in East Asia (pp. 21-40). Palgrave Macmillan, Cham. 
